\title{
Using the Virtual Cell simulation environment for extracting quantitative parameters from live cell fluorescence imaging data.
}

\author{
A. E. Cowan, L. Ye, F. R. Morgan, D. E. Koppel, B. M. Slepchenko, L. M. Loew and J. Schaff. \\ Center for Cell Analysis and Modeling, University of Connecticut Health Center, 263 Farmington \\ Ave., Farmington, CT 06030
}

Fluorescence probe and imaging technologies provide the means for highly detailed quantitative analysis of the temporal behavior of molecular interactions in cells. However it can be difficult to extract accurate parameters with simple image analysis tools because of the complex interplay of diffusion-reaction events with the morphology of the cell. As a result, only a small fraction of the available spatiotemporal information is utilized, and in many cases analysis remains at a qualitative level. The Virtual Cell simulation environment is uniquely suited to analyzing these types of fluorescence imaging experiments because it is designed to solve reaction-diffusion equations within any given geometry [1].

Virtual Cell (VCell; http://vcell.org/) is a problem solving environment for analysis, modeling and simulation of cell biological processes. VCell integrates a growing range of molecular mechanisms, including reaction kinetics, diffusion, flow, membrane transport, lateral membrane diffusion and electrophysiology, and can associate these with geometries derived from experimental microscope images. It is a web-based, client-server system, with more than two thousand world-wide users. The user interface allows experimentalists to input a physiological model in easily understood terms of species, reactions, and compartments. Segmented images are used to create a 2D or 3D geometry that matches the experimental cell for direct comparison. Development of the underlying math description and numerical methods to solve the simulation are transparent, although easily accessible for analysis and editing if desired. Furthermore, VCell can import almost any kind of generic images and proprietary microscopy data to be used within simulations to define arbitrary "fields" as the initial conditions of a simulation. Such fields can specify distributed parameters, initial conditions, or even $\mathrm{x}, \mathrm{y}, \mathrm{z}$ components of vector fields corresponding to e.g. cytoskeletal organization. Using VCell, a user can predict the spatiotemporal distribution of all molecular species given full information about initial distributions, model parameters, external stimuli, and the cellular geometry.

Many Virtual Cell models published by us and others were created specifically to simulate specific experimental situations, for example to analyze cellular effects of uncaging, experiments performed using total internal reflection optics, Fluorescence Loss in Photobleaching (FLIP) experiments to assess membrane association of the small GTPase rac, translocation of a GFP-tagged pleckstrin homology domain sensor for PIP2 and IP3, and Fluorescence Redistribution after Photobleaching (FRAP) studies of tight junction components (Shen et al., 2008) [2-6]. Although VCell is a powerful platform for analyzing imaging data, it is a general simulation tool and thus can be somewhat awkward to use for routine data analysis. We are therefore creating a suite of application-specific tools based on VCell technology to facilitate data analysis. This project, the "Virtual Microscopy Suite" will contain a series of applications, each directed at a single analysis mission, and they will be experiment-centric rather than model-centric. These tools will require that we develop new algorithms for directly comparing and optimizing simulations against the original experimental data. The first of these tools is VirtualFRAP, a downloadable executable designed to analyze FRAP 
experiments (http://ntenp.org/twiki/bin/view/VCell/VFRAP). It includes optimization algorithms for partial differential equation models and the ability to use experimentally-derived heterogeneous molecular distributions as initial conditions for simulation and parameter fitting. Currently, Virtual FRAP can be used to analyze experiments that collect all of the fluorescence associated with the cell to fit diffusion coefficients and \% recovery for either one or two diffusing components of cytosolic (soluble) proteins. We continue to develop VirtualFRAP and to create additional components of the Virtual Microscopy Suite. These include tools for the analysis of other photochemistry-based microscope manipulations such as (FLIP), uncaging or photoactivation experiments as well as for biosensors, , probe translocation, and Fluorescence Resonance Energy Transfer (FRET) experiments.

\section{References}

[1] B. M. Slepchenko et al., 2003. Trends Cell Biol. 13:570-6.

[2] P. Roy et al.. 2001. J Cell Biol. 153:1035-48.

[3] M. Holt,et al. 2004. Curr Biol. 14:173-83.

[4] K. Moissoglu et al. 2006. Mol Biol Cell. 17:2770-9.

[5] L. Shen et al. 2008. J Cell Biol. 181:683-95.

[6] Virtual Cell is developed by the National Resource for Cell Analysis and Modeling, supported by NIH P41RR13186; Virtual Frap is supported by U54RR022232.

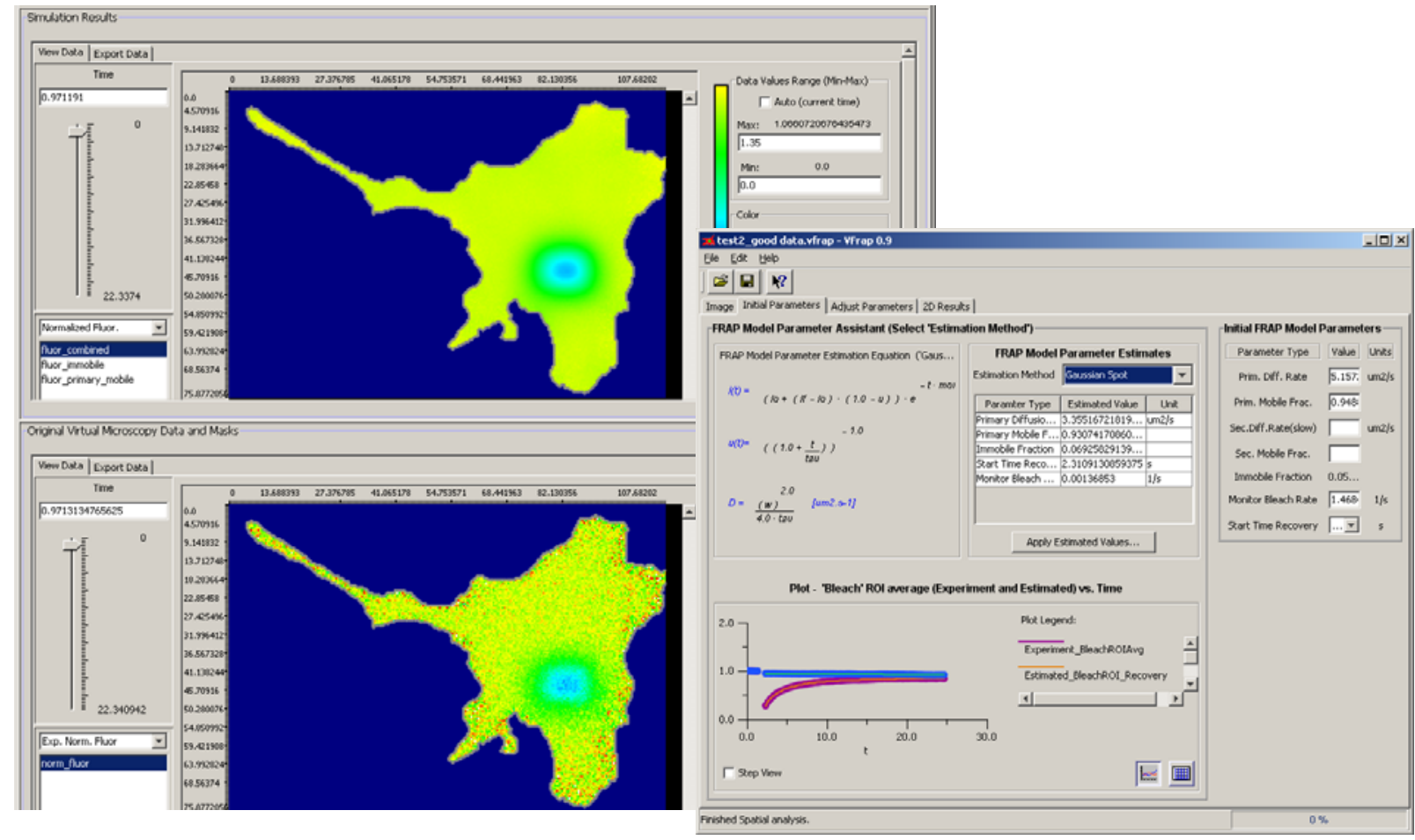

Figure 2. Comparison of the results of a Virtual Cell simulation of a fluorescence photobleaching experiment (top cell) to the actual experimental data (bottom cell), using the Virtual FRAP tool. 